Preprint, for final version see AIAA Journal, Vol. 54, No. 1, pp. 161-173, 2016. http://dx.doi.org/10.2514/1.J054123

\title{
Injection into Supersonic Boundary Layers
}

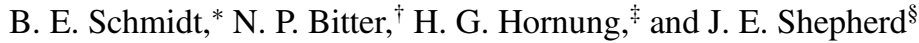 \\ California Institute of Technology, Pasadena, California 91125 \\ DOI: $\underline{10.2514 / 1 . J 054123}$
}

\begin{abstract}
A method for injection of gas into the boundary layer on a slender body in supersonic flow while minimizing perturbation to the mean flow is examined. Injection of gas is equivalent to a sudden increase in the displacement thickness of the boundary layer, which produces an oblique shock that propagates into the inviscid region of the flow. It is found that modification of the geometry of the body can compensate for the increased displacement thickness created by injection and minimize the production of oblique waves. However, the resulting near-wall injection layer is observed to be unstable and a turbulent boundary layer develops downstream of the injection region. The instability of the flow is examined experimentally using high-speed schlieren visualization and numerically using linear stability analysis of velocity profiles from a compressible Navier-Stokes computation. At the present postshock Mach number of about 3.8, both first- and second-mode instabilities are active, though computations predict that the first mode is primarily responsible for transition downstream of the injector.
\end{abstract}

\section{Nomenclature}

$\begin{array}{ll}L & =\text { length of injector, } \mathrm{m} \\ L_{c} & =\text { cone length, } 0.25 \mathrm{~m} \\ M & =\text { Mach number } \\ m & =\text { normalized injection mass flow rate } \\ \dot{m} & =\text { mass flow rate, } \mathrm{kg} / \mathrm{s} \\ p & =\text { pressure, } \mathrm{Pa} \\ R & =\text { specific gas constant, } \mathrm{J} /(\mathrm{kg} \cdot \mathrm{K}) \\ R e_{x} & =\text { Reynolds number based on } x \\ r & =\text { injector radial coordinate, } \mathrm{m} \\ r_{c} & =\text { local cone radius, } \mathrm{m} \\ T & =\text { temperature, } \mathrm{K} \\ U & =\text { velocity, } \mathrm{m} / \mathrm{s} \\ x & =\text { coordinate along cone surface, } \mathrm{m} \\ \alpha_{i} & =\text { spatial growth rate, normalized by } L_{c} \\ \beta & =\text { spanwise wavenumber, normalized by } L_{c} \\ \gamma & =\text { ratio of specific heats } \\ \delta & =\text { injection/boundary-layer thickness, } \mathrm{m} \\ \kappa & =\text { permeability, }{ }^{2} \\ \mu & =\text { dynamic viscosity, } \mathrm{kg} /(\mathrm{m} \cdot \mathrm{s}) \\ \omega & =\text { frequency, normalized by } L_{c} \text { and } U_{\infty} \\ \rho & =\text { density, kg/m } \mathrm{m}^{3}\end{array}$

Subscripts

$\begin{array}{lll}\infty & = & \text { freestream quantity } \\ e & = & \text { injection/boundary-layer edge quantity } \\ w & = & \text { wall quantity }\end{array}$

\section{Introduction}

NJECTION of a gas into the boundary layer in a supersonic flow has a number of applications to high-speed flight. In scramjet

Presented as Paper 2014-2496 at the 7th AIAA Theoretical Fluid Mechanics Conference, Atlanta, GA, 16-20 June 2014; received 30 December 2014; revision received 1 June 2015; accepted for publication 16 August 2015; published online 7 October 2015. Copyright (C 2015 by the American Institute of Aeronautics and Astronautics, Inc. All rights reserved. Copies of this paper may be made for personal or internal use, on condition that the copier pay the $\$ 10.00$ per-copy fee to the Copyright Clearance Center, Inc., 222 Rosewood Drive, Danvers, MA 01923; include the code 1533-385X/ 15 and $\$ 10.00$ in correspondence with the CCC.

*Graduate Student, Aerospace Engineering, 1200 E California Blvd. MC 205-45. Student Member AIAA.

${ }^{\dagger}$ Graduate Student, Aerospace Engineering, 1200 E California Blvd. MC 205-45. Fellow AIAA.

Frofessor, Aerospace Engineering, 1200 E California Blvd. MC 205-45. Fellow AIAA.

${ }^{\S}$ Professor, Aerospace Engineering, 1200 E California Blvd. MC 205-45. Member AIAA. inlets, injection can reduce skin friction drag, cool the wall by film cooling, increase the resistance of the boundary layer to separation via transition to turbulence, and mix some fuel with air before entering the combustor $[1,2]$. The motivation for the present study is the potential for instability control by the modification of boundarylayer gas composition. The concept is based on the observation that gases that are vibrationally active can convert acoustic energy into thermal energy, damping waves associated with boundary-layer instability and delaying transition to turbulence [3]. However, injection is also potentially destabilizing because of the modification of the boundary-layer velocity profile and the potential for adverse pressure gradients due to oblique shock wave generation. The present study does not consider the effects of vibrational nonequilibrium but only explores the fluid mechanics of supersonic flow with injection.

Hypervelocity boundary layers are highly unstable to acoustic disturbances via the so-called second or Mack mode [4]. The growth rates for the first mode, caused by Tollmien-Schlichting instability, are much smaller compared with that of the Mack mode when the freestream Mach number is high. The growth rate for the Mack mode is strongly dependent on the wall temperature, and when the wall is cold, as is usually the case in hypersonic flight, the Mack mode is dominant in controlling boundary-layer transition.

Current understanding of the mechanism for the Mack mode is summarized in Fedorov [5]. It is an inviscid instability brought about by trapped acoustic waves inside the boundary layer. The velocity and sound speed profiles in the boundary layer create a wave guide that refracts acoustic disturbances toward the wall and prevents them from escaping. The coupling of mean flow gradients with the trapped acoustic waves extracts energy from the mean flow, which reinforces and amplifies the fluctuations and eventually leads to turbulence. The line where the phase speed of the disturbance is equal to the sum of the flow velocity and the local sound speed, called the relative sonic line, acts as the upper boundary of the wave guide.

Past research has shown that acoustic energy can be absorbed by nonequilibrium effects in the gas in the boundary layer [ 6,7$]$. This occurs if two conditions are met: energy exchange between vibrational and translational/rotational modes must occur on the same time scale as the period of the most amplified frequency in the boundary layer, and the vibrational energy modes must be sufficiently populated to absorb a significant amount of acoustic energy. The most amplified frequency in hypervelocity boundary layers is on the order of $1 \mathrm{MHz}$. Both of these conditions are met for carbon dioxide at high-enthalpy flow conditions. For these reasons, if carbon dioxide could be injected into the boundary layer in a hypervelocity air flow without creating a significant disturbance, transition to turbulence would be delayed.

$\mathrm{CO}_{2}$ injection was previously tested in the $\mathrm{T} 5$ hypervelocity shock tunnel at Caltech by Leyva and Jewell $[\underline{3}, 8]$. These experiments were performed using a porous conical injector made from sintered stainless steel, the slope of which matches the slope of the cone 


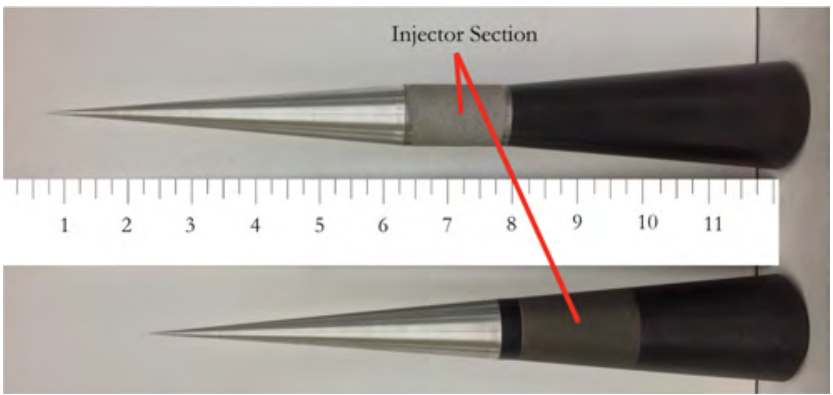

Fig. 1 Cylindrical (top) and conical (bottom) injector assemblies. Ruler units are inches.

model, shown in Fig. 1. One complicating factor in interpreting these experiments is that previous studies have shown that a porous surface with no injection absorbs acoustic energy and can significantly delay or suppress boundary-layer transition [9]. The results are inconclusive regarding the effectiveness of injection on delaying the onset of boundary-layer transition, motivating a closer study of the effects of gaseous injection in supersonic flow.

Upon further investigation, it is clear that injection from a conical injector creates a disturbance to the mean flow, which is undesirable. The injected gas can be seen as increasing the displacement thickness of the boundary layer over a short distance, which has the effect of a positive angle change of the surface of the cone. This causes an oblique shock to form that propagates into the exterior flow and creates an adverse pressure gradient on the surface, along with another wave system downstream related to the specifics of the injected gas flow.

It was hypothesized by A. Fedorov that a negative slope of the injector section could compensate for the increase in the displacement thickness caused by injection. This would minimize the strength of the oblique shock and other waves if the injection rate was properly tuned. Fedorov et al. [10] examined this hypothesis for T5 conditions with numerical simulations using an approach similar to that presented in Sec. IV.B. An illustration of this idea is shown in Fig. 2, and Fig. 1 shows the assembled model. On a cone with a cylindrical section without injection, it is well known that the boundary layer separates over the compression turn at the end of the cylindrical section. It will be investigated whether this occurs with injection.

\section{Experimental Setup}

Experiments for the current study were performed in the Ludwieg tube at Caltech [11]. A perspective view of the facility is shown in Fig. 3. The Ludwieg tube in its present configuration produces Mach 4 air flow with a freestream velocity of $670 \mathrm{~m} / \mathrm{s}$, a steady test time of $100 \mathrm{~ms}$, and a unit Reynolds number range of $5-15 \times 10^{6}$ per meter. The noise level is about $1 \%$ in pitot pressure. A standard Z-type schlieren visualization setup is used to visualize the flow. A Phantom v710 high-speed camera is used to record images, and two separate light sources are used. For sensitive, full-field images that clearly detect the shock system, a Cree X-Lamp MC-E Cool White continuous white light LED is used with a frame rate of 3000 frames per second and an exposure time of $30 \mu \mathrm{s}$. Images taken with this configuration cannot resolve small or transient structures in the flow, and so a second light source is used in a separate set of experiments with a different frame rate to capture these. The second source is an Osram SPL PL90-3 infrared laser diode with a wavelength of $905 \mathrm{~nm}$. The beam is expanded to achieve a field of view surrounding the injector, and the diode is pulsed according to the frame rate of the camera of 100,000 frames per second with a pulse width of $40 \mathrm{~ns}$ using a PicoLAS LDP-V 50-100 V3 laser driver. This allows resolution of

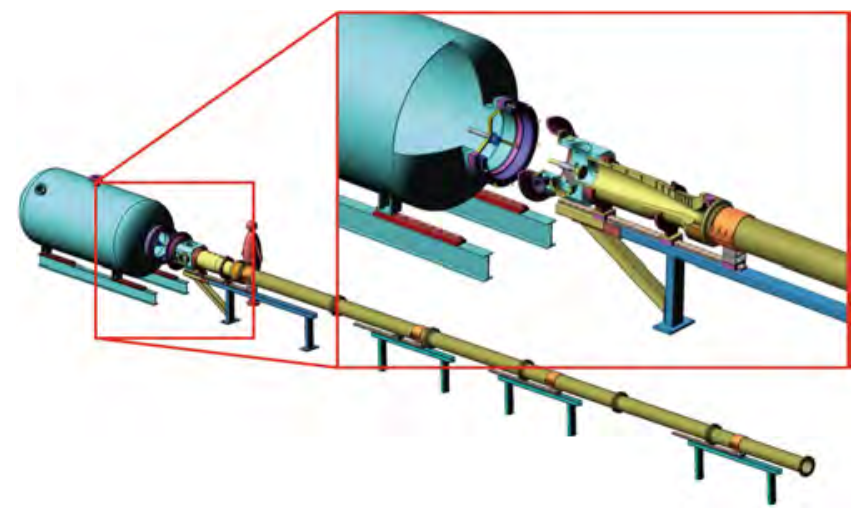

Fig. 3 Solid model of the Caltech Ludwieg tube.

small-scale or transient structures in the flow that cannot be observed using the continuous light source, but with the sacrifice of some spatial resolution. The pulsed diode technique in schlieren imaging is documented in [12].

Both the conical and cylindrical injectors used in this study are approximately $40 \mathrm{~mm}$ long with a diameter of $23 \mathrm{~mm}$ and are inserted between sections of a 5-deg half-angle cone. The beginning of the injector section is $127 \mathrm{~mm}$ from the tip of the cone. Both injectors are made from sintered $316 \mathrm{~L}$ stainless steel, and the cylindrical injector is observed to have a larger mean pore size than the conical injector and therefore has a higher permeability. Figure 4 shows $5 \times$-magnified images of the surface of each injector, clearly illustrating the larger pores on the cylindrical injector. The permeability of each is calculated in Sec. III.

The mass flow rate of injected gas is measured with a Sensirion EM1 mass flow meter and is presented as a parameter $m$, which is the injected mass flow rate normalized by the mass flow rate of the incoming laminar boundary layer based on $\delta_{99}$. The EM1 is calibrated for air by the manufacturer with a rated accuracy of $\pm 5 \%$ and was calibrated for carbon dioxide using a King Instruments rotameter. The incoming boundary-layer profile used for calculation of $m$ is calculated using the Taylor-Maccoll solution for supersonic flow over a cone coupled with the similarity solution of Lees for a laminar compressible boundary layer on a cone. The boundary layer is observed with high-speed schlieren imaging to be laminar before the injector in all configurations. The calculated laminar velocity and density profiles are multiplied and integrated from zero to $\delta_{99}$ to calculate the mass flow rate. $\delta_{99}$ in this case is $0.75 \mathrm{~mm}$ and the mass flow rate of gas in the incoming boundary layer is calculated to be $0.80 \mathrm{~g} / \mathrm{s}$. The unit Reynolds number based on freestream quantities, $R e_{\infty}=\rho_{\infty} U_{\infty} / \mu_{\infty}$, is approximately $10^{7} / \mathrm{m}$, corresponding to a Reynolds number based on $x, R e_{x}=\rho_{\infty} U_{\infty} x / \mu_{\infty}$, where $x$ is the coordinate along the cone surface, of $1.27 \times 10^{6}$ at the beginning of the injector.

\section{Porous Media Analysis}

Before examining the results of the experiments it is worthwhile to analyze the flow through the porous injectors. A more complete discussion of the flow through the injectors can be found in a report by Schmidt [13]. Only the relevant results are reported here. The flow through an injector is assumed to be axisymmetric and steady, and a relationship between the pressure inside the injector and the mass flow rate through the injector can be derived from the continuity and momentum equations. The conservation of mass for steady flow in the radial direction through the injector is
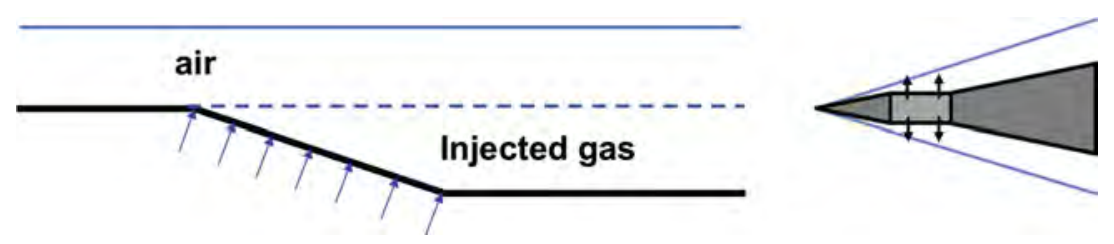

Fig. 2 Illustration of the concept of a negatively-sloped injector. 


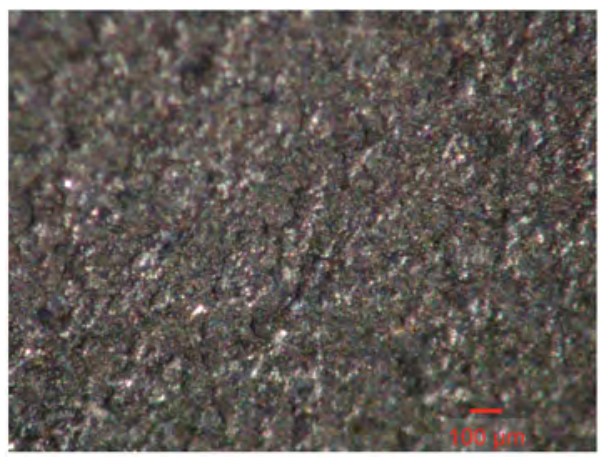

a) Conical injector

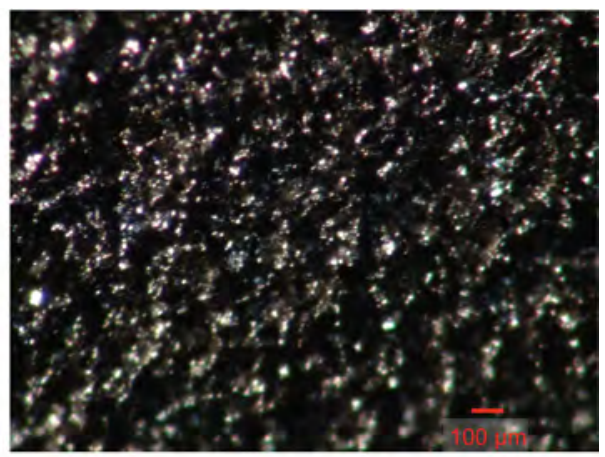

b) Cylindrical injector

Fig. 4 Five-times-magnified images of the surface of injectors.

$$
\rho u r=\text { constant }=\frac{\dot{m}}{2 \pi L}
$$

for a cylinder of length $L$. The momentum equation is a balance between the pressure difference across the injector and the drag inside the pores modeled with Darcy's law for porous flow

$$
-\frac{\mathrm{d} p}{\mathrm{~d} r}=\frac{\mu u}{\kappa}
$$

Here $\kappa$ is the permeability of the porous material. Substituting the mass flow rate for velocity according to Eq. (1), the following relation between pressure and mass flow rate is derived:

$$
-\rho \frac{\mathrm{d} p}{\mathrm{~d} r}=\frac{\dot{m} \mu}{2 \pi L \kappa r}
$$

Using the ideal gas law to express $\rho$ as a function of $p$ and $T$ allows this equation to be integrated from the inside of the injector to the outside approximating the gas as isothermal through the injector, which is reasonable because the local Mach number remains small. This integration yields

$$
p_{i}^{2}-p_{o}^{2}=\frac{\dot{m} \mu R T}{\pi L \kappa} \ln \left(\frac{r_{o}}{r_{i}}\right)
$$

where subscripts $i$ and $o$ denote conditions at the inside and outside of the injector, respectively. In the experiments, the pressure at the outside of the injector is the boundary-layer edge pressure for the cone, which can be computed according to the Taylor-Maccoll solution to be $1.73 \mathrm{kPa}$. The pressure required to drive an appreciable mass flow through the injector, as can be seen from Figs. $5 \mathrm{a}$ and $5 \mathrm{~b}$, is on the order of $100 \mathrm{kPa}$. Thus, $p_{o}^{2} / p_{i}^{2} \ll 1$ and Eq. (4) can be approximated as

$$
p_{i}^{2}=\frac{\dot{m} \mu R T}{\pi L \kappa} \ln \left(\frac{r_{o}}{r_{i}}\right)
$$

A set of experiments was performed to simultaneously measure the pressure inside the injector and the mass flow rate in order to confirm the validity of Eq. (5) and determine the value of the permeability of each injector. A measurement unit fitted with a Kulite XT-190 piezoresistive pressure transducer was fitted to the front of the injector instead of the cone tip used in flow experiments, and the apparatus was placed inside the test section of the Ludwieg tube at a partial vacuum of approximately $1.7 \mathrm{kPa}$ to simulate the proper edge pressure that would occur during a flow experiment.

Figure 5 shows the results of these experiments with air as the injected gas for each injector. The permeability $\kappa$ is determined by linear least squares by considering the relationship between pressure and the square root of the mass flow rate. It can be seen from Eq. (5) that $\kappa$ is the only unknown parameter, and so it is determined by the fit that minimizes the total error. The permeability of the cylindrical injector is $3.5 \pm .003 \times 10^{-12} \mathrm{~m}^{2}$ and the permeability of the conical injector is $1.1 \pm .0006 \times 10^{-14} \mathrm{~m}^{2}$. Uncertainty in $\kappa$ is calculated by the quadrature sum of the uncertainty based on the $95 \%$ confidence interval of the curve fit and the propagation of uncertainties in pressure and mass flow rate. The calculated values for permeability are typical for sintered metal [14].

\section{Results and Discussion}

Tests in supersonic flow are performed across a wide range of injection mass flow rates with both injectors. Although carbon dioxide would eventually be the injected gas in a boundary-layer transition control experiment in hypervelocity flow, both carbon dioxide and air were used as the injected gas in the current study. Injecting air simplifies computational analysis of the flow because the mass transport equation decouples from the Navier-Stokes equations. At room temperature, the temperature at which the gas is injected, carbon dioxide is not significantly vibrationally active, and so injection of gases with such similar density is not expected to qualitatively alter

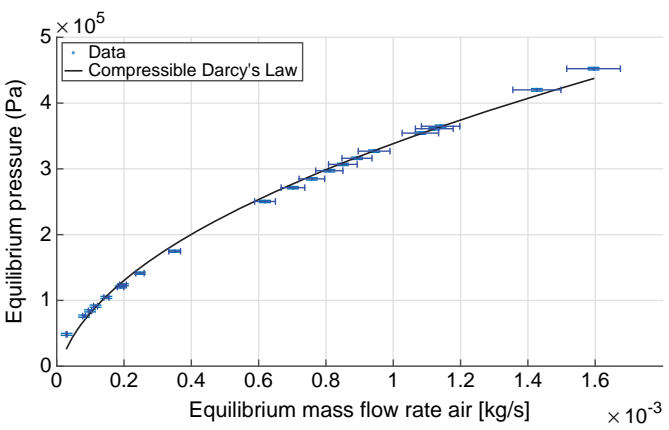

b) Conical injector

a) Cylindrical injector

Fig. 5 Pressure versus mass flow rate data for air for both injectors fit with Eq. (ㅁ) to determine permeabilities. 


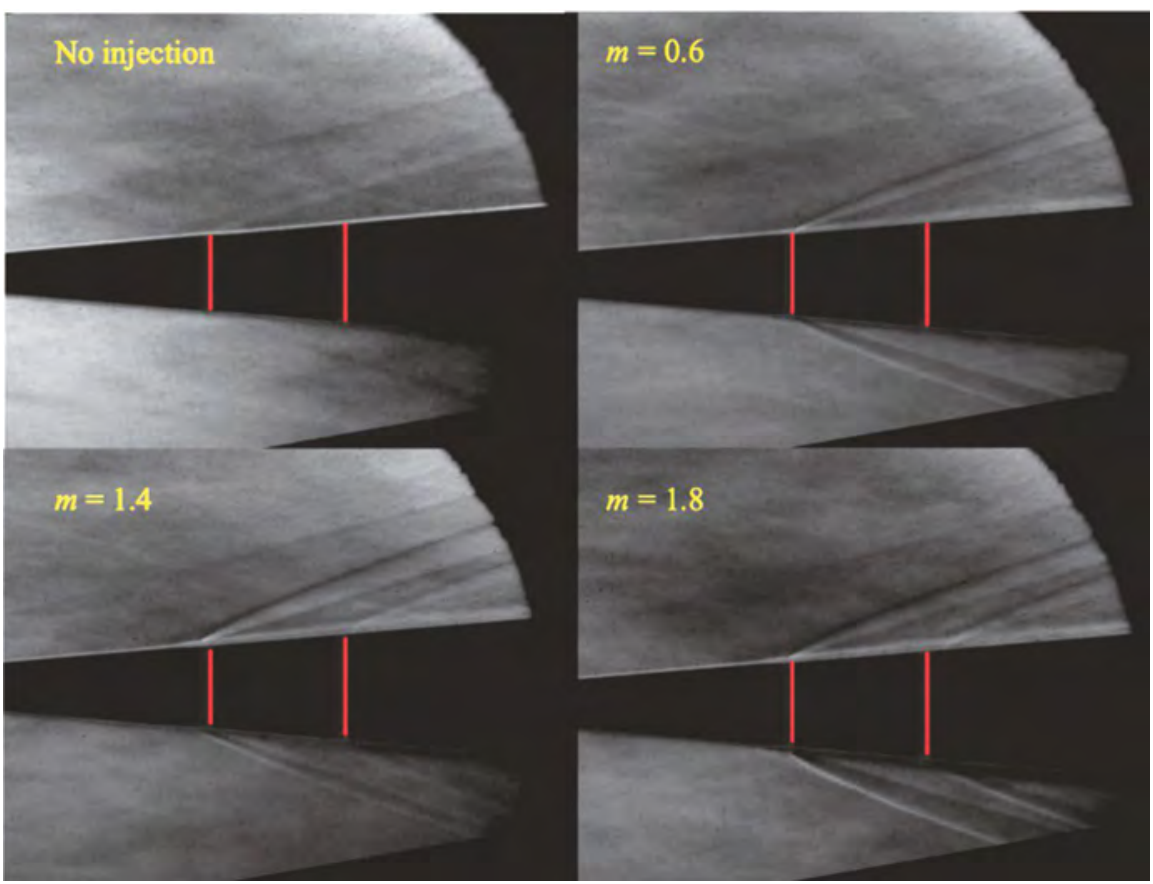

Fig. 6 Long-exposure schlieren images from conical injector experiments using air.

the flow characteristics. This assumption is shown to be valid in a set of experiments performed with carbon dioxide as the injected gas.

\section{A. Experiment}

Schlieren images from four tests with the conical injector are shown in Fig. 6. The vertical lines on the cone model mark the location of the injector, and the parameter $m$ again represents the normalized mass flow rate described in Sec. II. A stronger shock, relative to the shock at the cone tip, is visible in all three injection tests at the beginning of the injector due to the increased displacement thickness as predicted in Sec. I. The shock angle at the cone tip is determined to be $15 \pm 0.1^{\circ}$ both by experiment and via the TaylorMaccoll solution, which corresponds to a nondimensional pressure jump of $\Delta p / p_{1}$ of $0.084 \pm 0.016$ by perfect-gas shock relations. The shock angle at the beginning of injection is measured to be approximately $21 \pm 0.1^{\circ}$ relative to the cone surface, corresponding to a pressure jump of $\Delta p / p_{1}$ of $1.00 \pm 0.02$, or about 13 times stronger than the shock at the cone tip. The shock created by injection is curved, and so it is difficult to precisely determine its angle at the surface of the cone, and the angle is $21^{\circ}$ within experimental uncertainty for all injection mass flow rates studied. Besides appreciably disturbing the inviscid region of the flow, the oblique shock also creates a strong adverse pressure jump for the incoming boundary layer, potentially leading to separation or rapid transition to turbulence. An expansion wave appears in the region behind this shock, and a second oblique shock wave is visible at the end of the injector in tests with high mass flow rates. This wave system is what one would predict by modeling the additional displacement thickness over the injector as a convex bump on the surface of the cone.

Figure 7 shows the results from four representative tests with the cylindrical injector. Three regimes are possible in this configuration. In cases with high mass flow rates, represented by $m=1.0$ and

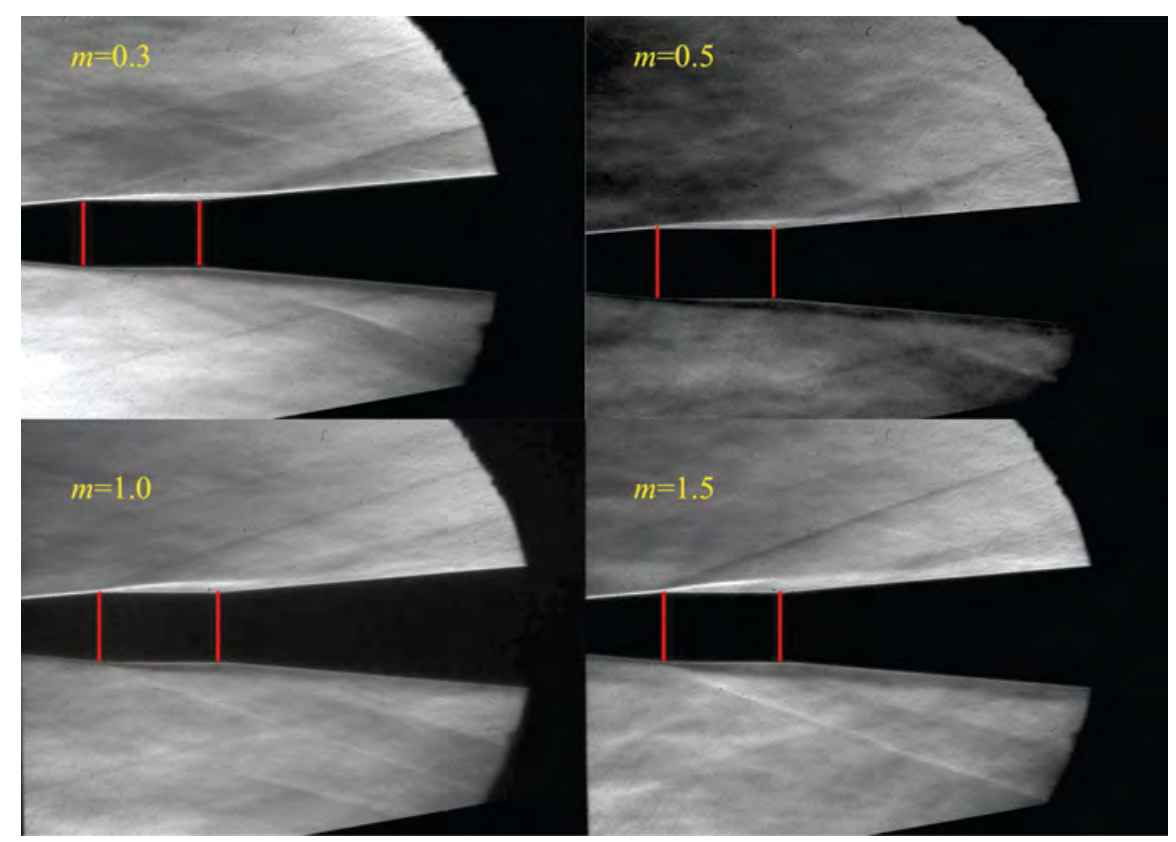

Fig. 7 Long-exposure schlieren images from cylindrical injector experiments using air. 


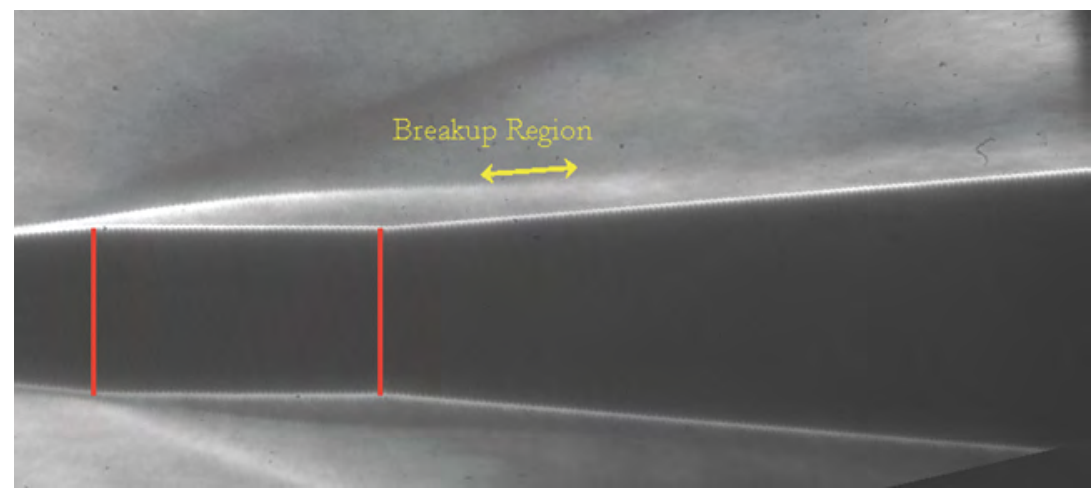

Fig. 8 Example schlieren image used to determine injection layer transition location. Transition location is clear from the breakup at the outer edge of the layer.

$m=1.5$, an oblique shock is still formed at the beginning of the injector as the additional displacement thickness from injection overcompensates for the negative slope of the injector itself. If the mass flow rate is sufficiently small, such as $m=0.3$, the injection is not large enough to compensate for the shape change of the injector, resulting in a weak expansion wave at the beginning of the injector. However, at a normalized mass flow rate of approximately $m=0.5$, the slope from the increasing displacement thickness very nearly matches the slope of the cone and no visible waves are observed to propagate into the free stream. The mean flow is therefore closely matched to flow past a cone with no injection and no changes to its geometry. These results validate the hypothesis of Fedorov and demonstrate that it is possible to inject gas in the boundary layer around a body with a minimal impact on the exterior supersonic flow.

For the specific application of influencing boundary-layer transition, the key issue is the effect of injection on boundary-layer stability: does the boundary layer transition rapidly from laminar to turbulent, or does it remain laminar downstream? Previous work by Pappas and Okuno [15,16] and others [17] reveal that injection on a cone causes transition to occur earlier than with no injection in cold supersonic flow with lighter injected gas having a more pronounced effect [17]. Shaping of the injector has not been considered in previous studies, and so our results are unique in that regard.

Mean transition locations can be determined from high-speed schlieren images like the one shown in Fig. 8. The transition location is defined as the position where the injection layer edge ceases to be a sharp change in contrast in the image and becomes a smooth gradient broadening in extent with increasing downstream distance. Transition is observed to occur immediately at the beginning of the injector with the conical injector. This result differs from that of many other researchers [17,18], likely because of the particular injector being used in the present study. The injector is considerably smaller in area than those used in other studies, and so the injection rate per unit area is about an order of magnitude higher. This would create a stronger shock wave at the leading edge of the injector, possibly causing bypass transition associated with the large pressure jump across the shock. The precise cause will be examined further in future work.

Transition does not occur immediately with the cylindrical injector, however. Even at the highest mass flow rate tested $(m=3.8)$, the angle of the shock wave created by injection is $18 \pm 0.1^{\circ}$ relative to the cone surface, which is a pressure jump of $0.442 \pm 0.017$, about half of that created by injection with a conical injector. This may explain why bypass transition is not observed with the cylindrical injector. Figure 9 shows transition Reynolds number (based on freestream parameters) plotted versus normalized mass flow rate for both carbon dioxide and air injection. Error bars in transition location are determined from two factors: the width of the breakup region illustrated in Fig. 8 and unsteadiness in transition location observed in movies of images like that in Fig. 8. Transition location is unsteady due to perturbations in the tunnel freestream. In certain cases in which air is the injected gas, uncertainties are larger because of decreased contrast in the images compared with carbon dioxide cases. The decreased contrast makes determining transition location more difficult, particularly for cases with lower injection rates. Unsteadiness in the freestream also seems to have a more pronounced effect of changing transition location for low-mass flow cases with air. The trends in Fig. 9 agree qualitatively with those of Pappas and Okuno; namely, transition length decreases with increasing injection rate, and the trend appears to be more pronounced for air than for carbon dioxide, which also agrees with the findings of Pappas and Okuno on

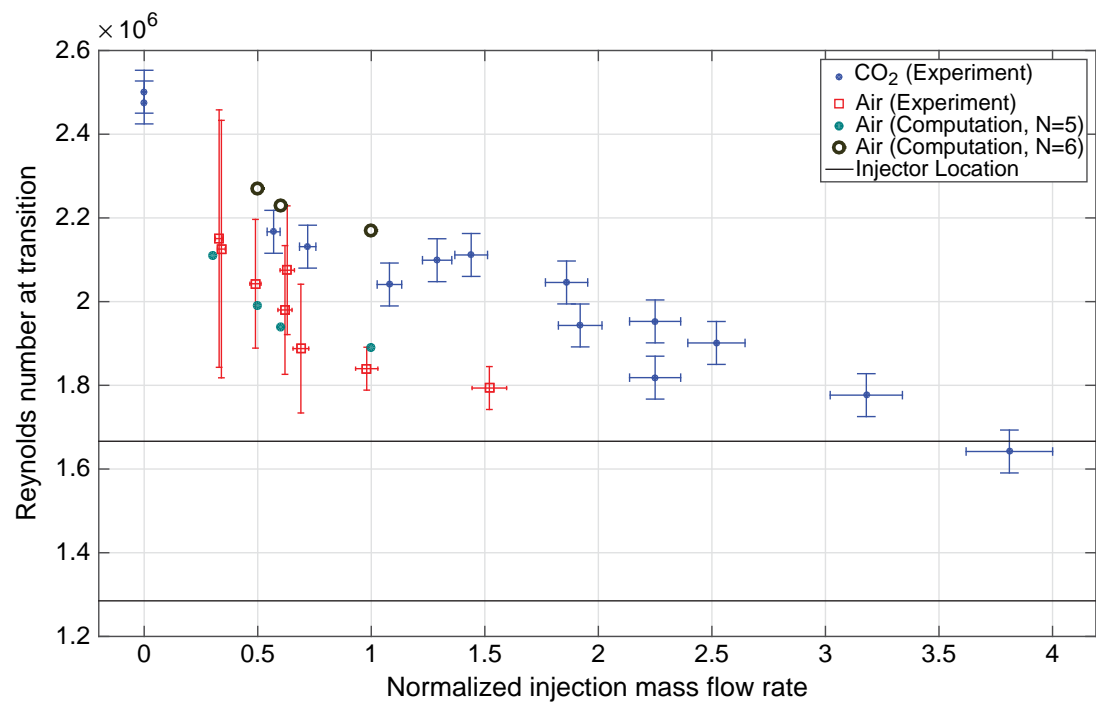

Fig. 9 Experimental and computational mean transition Reynolds number $\left(R e_{\mathrm{tr}}=\rho_{\infty} U_{\infty} x_{\mathrm{tr}} / \mu_{\infty}\right)$ for various injection rates with the cylindrical injector. Transition is observed near the end of the viewing window with zero injection. 


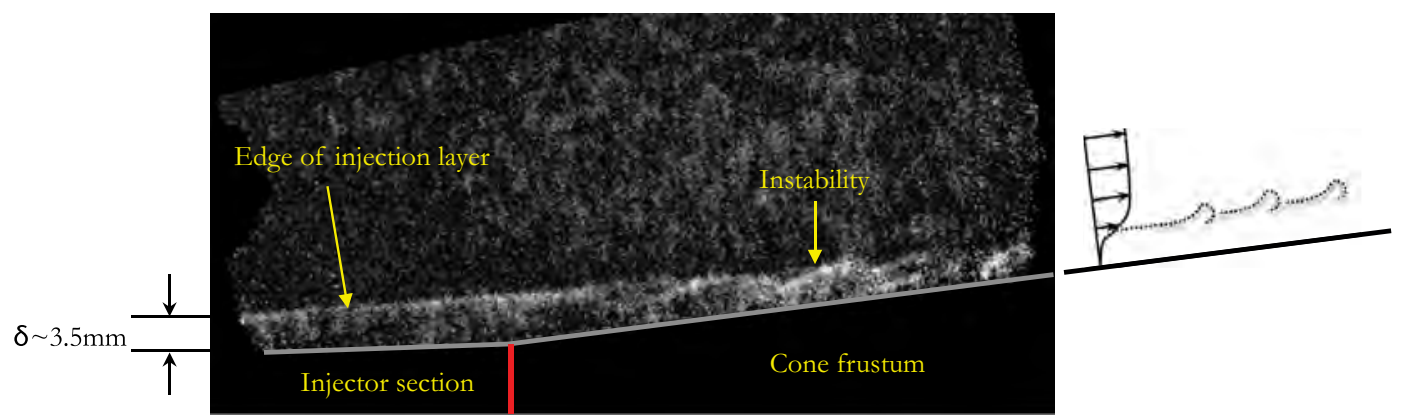

Fig. 10 Schlieren image from a test with $40 \mathrm{~ns}$ exposure with $m=0.6$.

the effect of injected gas density [15]. This effect is due at least in part to the higher injection velocity for lighter gases for a given mass flow rate. Computed transition Reynolds numbers following the analysis in Sec. IV.B agree with the experimental data for values of $N$ between 5 and 6.

Figure 10 shows an image taken with the pulsed laser source revealing regular turbulent structures forming downstream of injection. The structures appear to be very similar to those noted by Pappas and Okuno [16] but the nature of the instability is unclear. The sketch on the right of Fig. 10 shows the velocity profile and the resulting production of vorticity. The laser source also reveals that with injection the boundary layer does not appear to separate over the compression turn at the aft end of the injector.

Two cases with different injection mass flow rates are examined with pulsed laser schlieren imaging. The $40 \mathrm{~ns}$ exposure time freezes the motion of the instability waves and spatial wavelengths can be extracted. Each case contains 4000 images captured at 100,000 frames per second. Each image is 152 by 320 pixels, and the field of view is from $x=151 \mathrm{~mm}$ to $x=200 \mathrm{~mm}$ measured from the cone tip. An algorithm processes the images as follows. Each image is first filtered using pixelwise adaptive Wiener filtering with 3 by 3 neighborhoods to remove excess noise. Pixel intensities are sampled on a line 231 pixels $(42.4 \mathrm{~mm})$ long parallel to the cone edge at

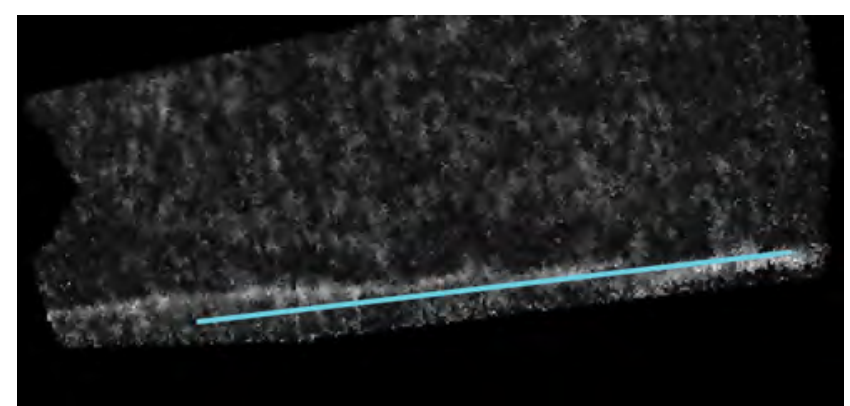

Fig. 11 Smoothed single image from case with $m=0.6$ with line representing location of samples for spectral analysis. varying wall-normal distances. Figure 11 shows a filtered image from

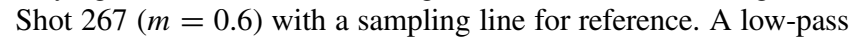
filter is then applied to the sampled data with a spatial frequency cutoff of 2 per mm, which further suppresses pixel-to-pixel variations. The peak spatial frequency can be shown to be insensitive to the cutoff if it is greater than 0.5 per mm. Welch's method with 8 windows with $50 \%$ overlap is used to estimate the power spectral density (PSD) of the pixel intensities along the sample line. The resulting PSD is interpolated from 129 points to 1000 using cubic spline interpolation to smooth the result at low spatial frequencies (long wavelengths).

The resulting 4000 spectra fall into three general categories, which are illustrated in Fig. 12. The majority of images contain no welldefined wavelength and therefore have a peak at zero wavenumber in their spectra, as shown in Fig. 12a. Some images have spectra containing a nonzero peak in wavenumber but also have significant lowfrequency noise, as shown in Fig. 12b. The signal-to-noise ratio in these images is therefore not considered to be high enough to retain them for spectral analysis. Approximately $20 \%$ of the images, however, have spectra like that in Fig. 12c, exhibiting a well-defined nonzero peak in wavenumber substantially above the low-frequency noise. Spectra are rejected if they have a peak at zero wavenumber or if the power density at the peak is less than twice as high as the power density at zero wavenumber. The spectra that are retained for analysis are averaged to give final spectra like those shown in Fig. 13.

A final averaged PSD is recorded for each sampling line location. The peak wavelength at each wall-normal distance is shown in Fig. 14. The sizes of the circular markers on the image correspond to the intensity of the peak wavelength. From analyzing the data the average thickness of the layer can be determined, it is slightly greater than $3 \mathrm{~mm}$ for $m=0.6$ and about $6 \mathrm{~mm}$ for $m=1.7$. Figure 14 demonstrates that the wavelength determined by the image processing algorithm is not sensitive to the location of the sampling line over the majority of the injection layer. Figure 13 shows the averaged PSD for the location with the highest peak intensity for each case, which is $1.47 \mathrm{~mm}$ from the wall for $m=0.6$ and $3.86 \mathrm{~mm}$ from the wall for $m=1.7$. The peak wavelengths are $6.8 \mathrm{~mm}$ for $m=0.6$ and $6.6 \mathrm{~mm}$ for $m=1.7$. It is interesting to note that the dominant instability in the layer has the same wavelength within experimental

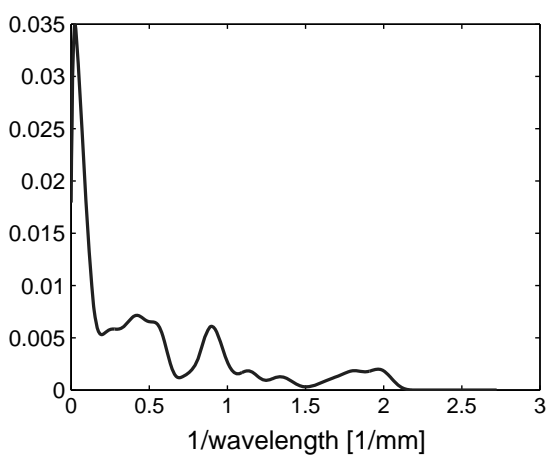

a) PSD with a peak at zero wavenumber

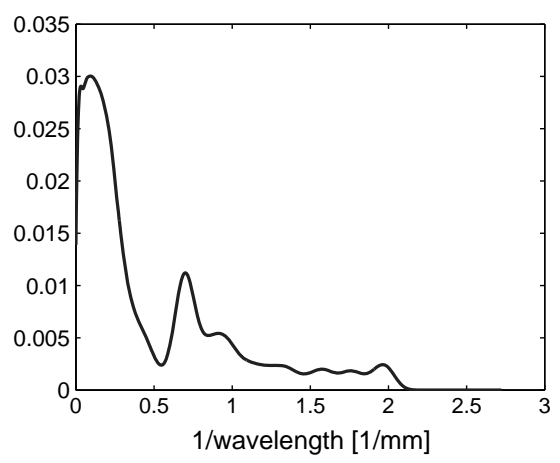

b) PSD with a nonzero peak but low-frequency noise

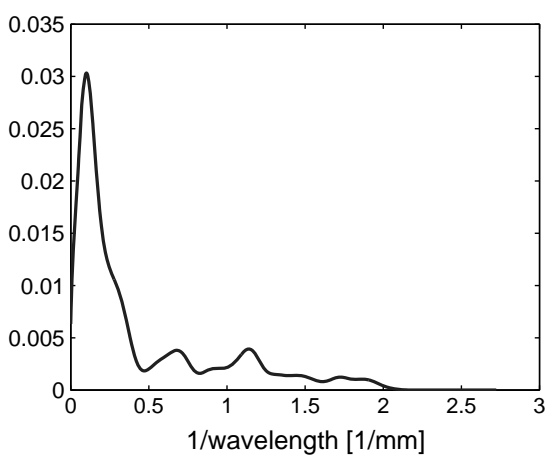

c) PSD with a well-defined nonzero peak

Fig. 12 Example spectra from case with $m=0.6$. 


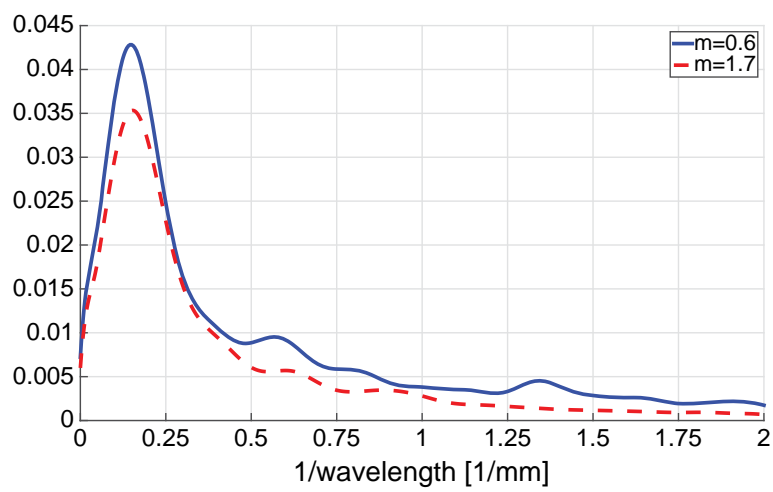

Fig. 13 Averaged PSDs for cases with $m=0.6$ and $m=1.7$.

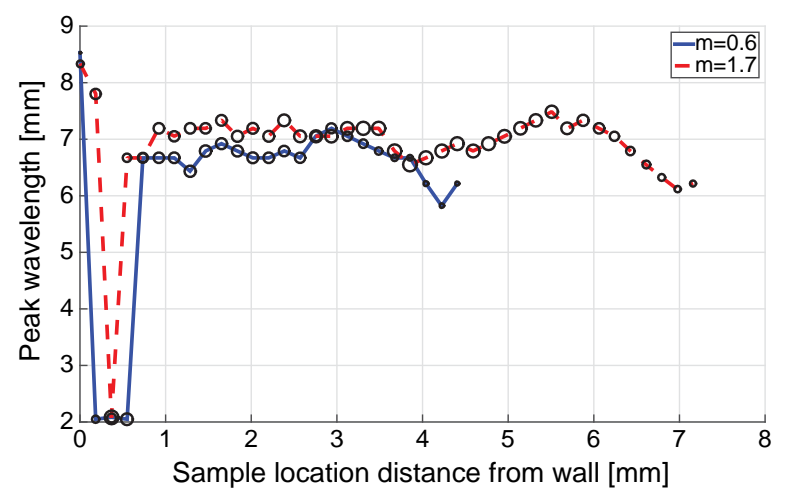

Fig. 14 Peak wavelengths for each case. The sizes of the circular markers correspond to the strength of the peak.

uncertainty in both cases even though the injection layer is approximately twice as thick in the $m=1.7$ case as the $m=0.6$ case. This behavior is not characteristic of second- or third-mode acoustic boundary-layer instabilities.

There is a short-wavelength wave present near the wall that is not present elsewhere in both cases. Spectra from sampling lines close to the wall can be examined to investigate this phenomena. Figure 15 shows three spectra from sampling lines nearest the wall for the $m=0.6$ case and Fig. 16 is the same for the $m=1.7$ case. These spectra show that a short-wavelength disturbance with a wavelength of approximately $2 \mathrm{~mm}$ is dominant nearest the wall, but as one moves away from the wall the strength of the short-wavelength disturbance is rapidly reduced while the longer-wavelength disturbance that is dominant elsewhere in the layer grows in amplitude. Interestingly, the short-wavelength disturbance has the same wavelength for both cases just like the long-wavelength disturbance.

\section{B. Stability Calculations}

The stability of the injection layer directly above and downstream of the porous surface was explored using linear stability analysis. The mean boundary-layer profile was computed using a shock-capturing Navier-Stokes solver that is included in the STABL stability software suite, developed at the University of Minnesota [19-21]. This program uses the data parallel line relaxation method to obtain steady-state solutions of the Navier-Stokes equations. Simulations were carried out for both the cone and cone-cylinder geometries described in Sec. II. For the conical geometry, a mesh of 400 streamwise and 420 wall-normal cells was used, while for the cone-cylinder geometry 1450 streamwise and 650 wall-normal cells were used. In both cases the grid points were clustered vertically near the wall and also horizontally over the injector. For the cylindrical injector, simulations were also carried out with a coarser mesh of $850 \times 450$ cells and no significant differences were seen either in the mean boundary layer or in the stability calculations, indicating that the mesh is converged.

The prescribed freestream conditions match those of the Ludwieg tube. The freestream velocity is $670 \mathrm{~m} / \mathrm{s}$, the pressure is $1335 \mathrm{~Pa}$, and the temperature is $70 \mathrm{~K}$. The injector is modeled using a fixed mass flux with an injection temperature of $300 \mathrm{~K}$. Both the freestream and injected gas are air, which is modeled as a calorically perfect ideal gas. Examples of computed density contours for several different injection rates are shown in Fig. 17, in which injection rates are chosen to match those of the experiments in Figs. $\underline{6}$ and 7 . The simulated wave structures are in good qualitative agreement with the schlieren photographs. For the conical injector, all values of $m$ produce a shock at the leading edge of the injector because of the increased displacement associated with injection. For the conecylinder geometry, the leading edge of the injector produces an expansion wave for $m<0.5$ and a shock wave for $m>0.5$, while at $m \sim 0.5$ these wave structures are nearly eliminated.

Figure 18 shows velocity and temperature profiles for the cylindrical injector with $m=0.5$. The first profile in this sequence matches the self-similar profile, unaffected by injection. Over the injector, $127-167 \mathrm{~mm}$, the boundary-layer thickness increases by about a factor of four and the velocity profile develops an inflection point. For large injection rates, the inflection in the velocity profile grows narrower and the velocity profile begins to resemble that of a shear layer. Downstream of the injector the velocity and temperature profiles slowly relax back toward self-similarity, though they do not relax fully by the time the end of the cone is reached.

At each streamwise location $x$, the base flow from the NavierStokes simulation is interpolated onto a wall-normal mesh and a locally parallel stability analysis is conducted using the shooting method developed by Mack [22]. Perturbations of the form $\boldsymbol{q}(y) \exp (i \alpha x+i \beta z-i \omega t)$ are substituted into the linearized Navier-Stokes equations, where $\alpha$ and $\beta$ are the streamwise and spanwise wavenumbers, $\omega$ is the frequency, and $\boldsymbol{q}$ is the vector of complex disturbance amplitudes:

$$
\boldsymbol{q}=\left(\hat{u}, \frac{\mathrm{d} \hat{u}}{\mathrm{~d} y}, \hat{v}, \hat{p}, \hat{\theta}, \frac{\mathrm{d} \hat{\theta}}{\mathrm{d} y}, \hat{w}, \frac{\mathrm{d} \hat{w}}{\mathrm{~d} y}\right)^{T}
$$

The frequency and wavenumbers are nondimensionalized using the cone length, $L_{c}=0.25 \mathrm{~m}$, as the length scale and the convection time, $L_{c} / U_{e}$, as the characteristic time scale. In terms of the

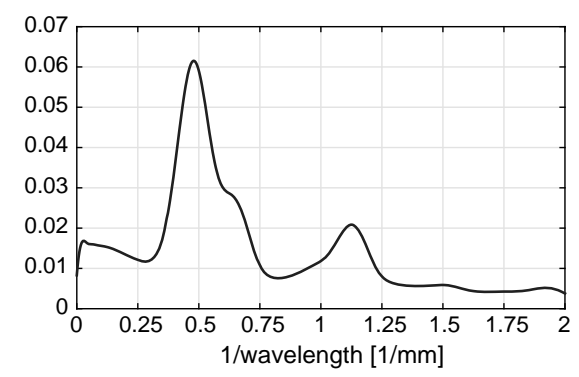

a) $0.37 \mathrm{~mm}$ from wall

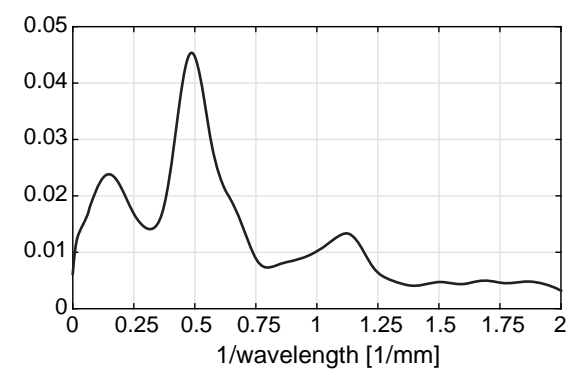

b) $0.55 \mathrm{~mm}$ from wall

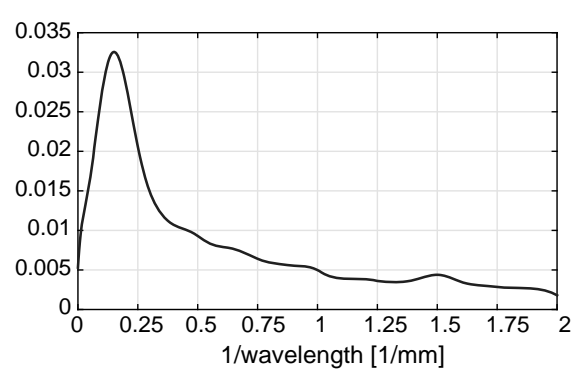

c) $0.73 \mathrm{~mm}$ from wall

Fig. 15 Near-wall spectra from case with $m=0.6$. 


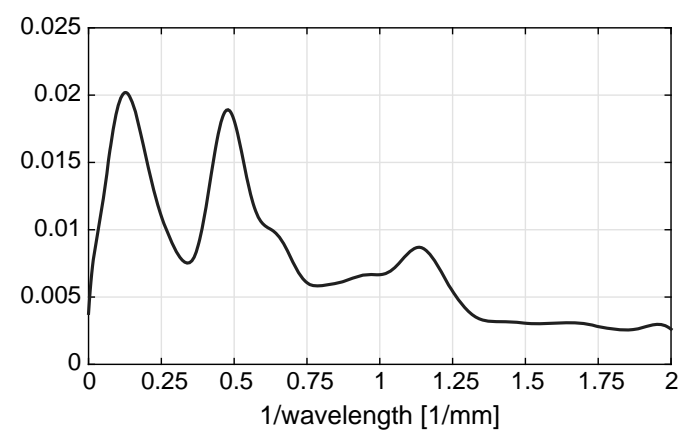

a) $0.18 \mathrm{~mm}$ from wall

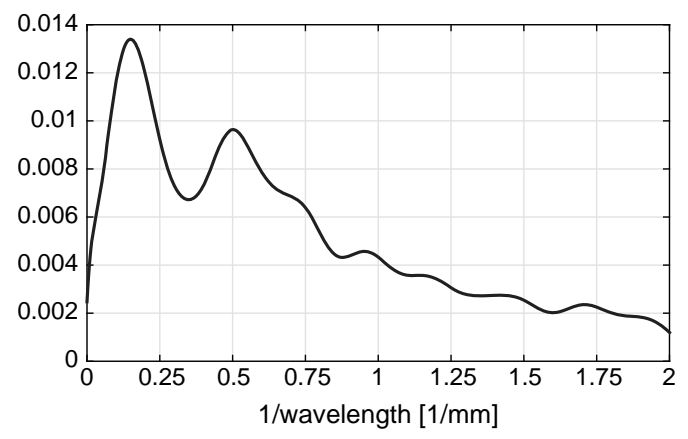

a) $0.55 \mathrm{~mm}$ from wall

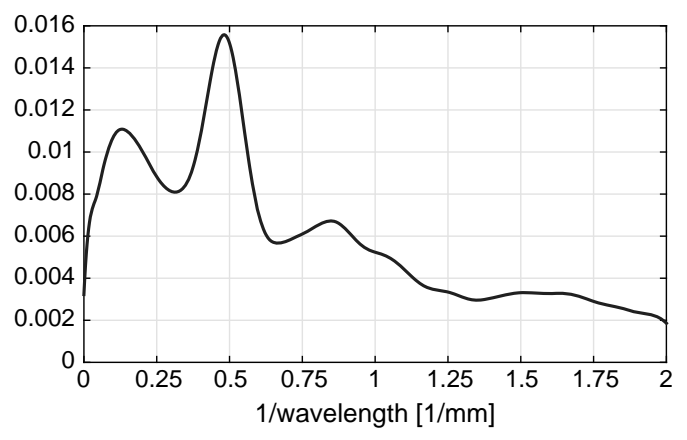

b) $0.37 \mathrm{~mm}$ from wall

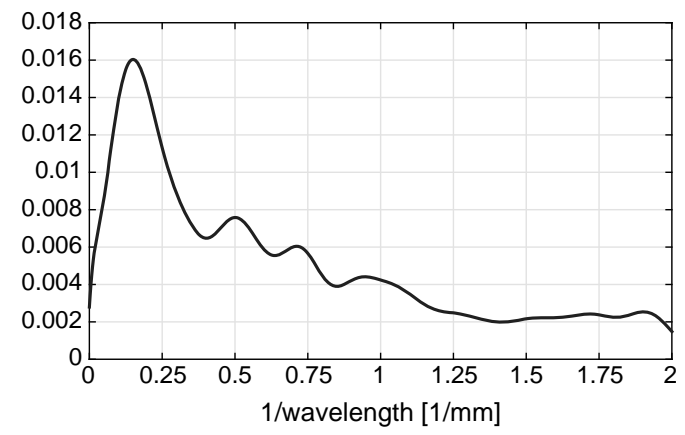

b) $0.73 \mathrm{~mm}$ from wall

Fig. 16 Near-wall spectra from case with $m=1.7$.
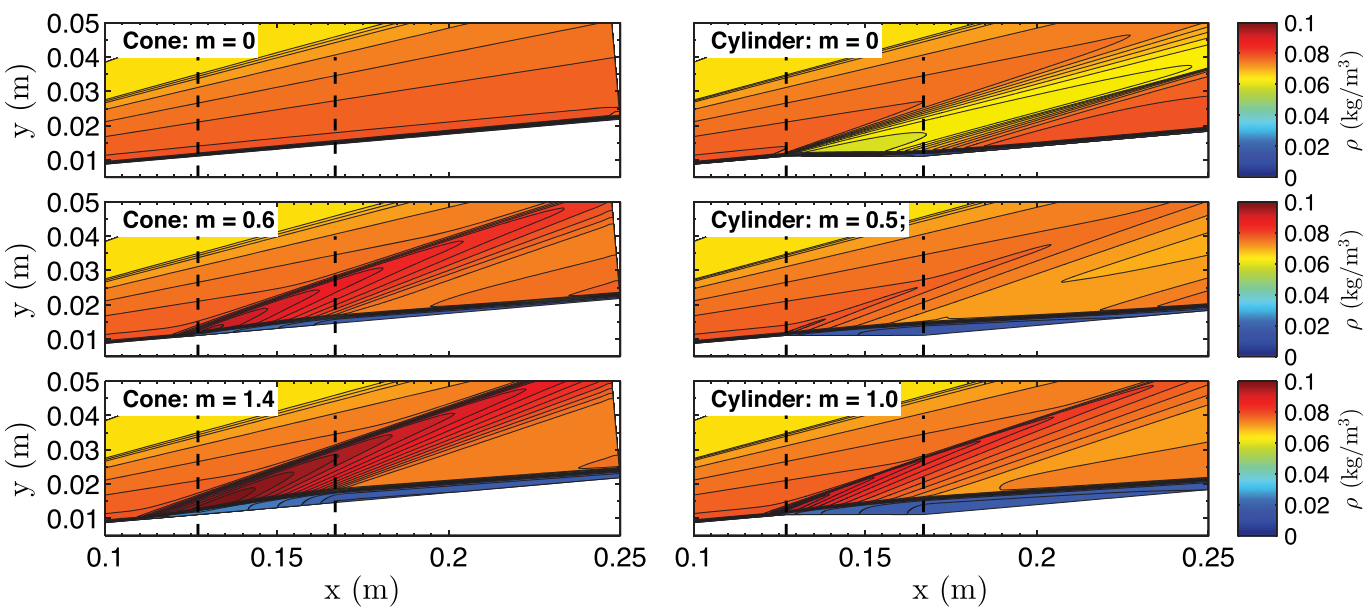

Fig. 17 Density contours for several different injection rates and injector geometries. Vertical dashed lines designate injector boundaries. Left column, conical injector; right column, cylindrical injector.

disturbance variables above, the linearized Navier-Stokes equations can be cast in the form:

$$
\frac{\mathrm{d} \boldsymbol{q}}{\mathrm{d} y}=\boldsymbol{A q}
$$

where $\boldsymbol{A}$ is an $8 \times 8$ matrix whose coefficients are given by Malik [23]. This system of equations is solved starting in the freestream where the asymptotic behavior as $y \rightarrow \infty$ is known (see [22]). In the freestream there are eight fundamental solutions of Eq. (7), but because the disturbances must be decaying as $y \rightarrow \infty$, only four of them are admissible. These four admissible (decaying) fundamental solutions are then integrated toward the wall using a fourth-order Runge-Kutta routine. Gram-Schmidt orthonormalization is employed intermittently as this integration proceeds to control parasitic growth of numerical errors [24-26]. Linear combinations of the four decaying solutions of Eq. (7) are taken at the wall to satisfy the homogeneous velocity boundary conditions $\hat{u}=\hat{v}=\hat{w}=0$ and the normalization condition $\hat{p}=1$, while the remaining homogeneous
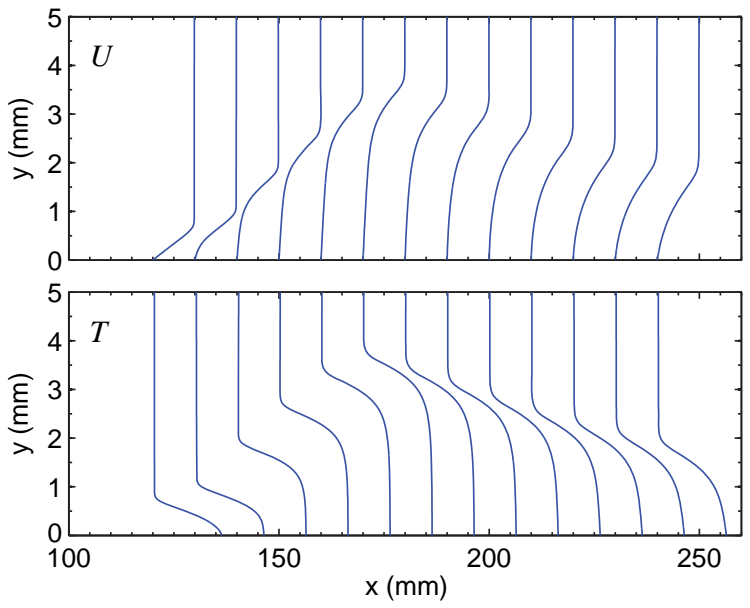

Fig. 18 Velocity (top) and temperature (bottom) profiles for the cylindrical injector, $m=0.5$. The injector extends from $x=127-167 \mathrm{~mm}$. Profiles are taken along wall-normal planes. 

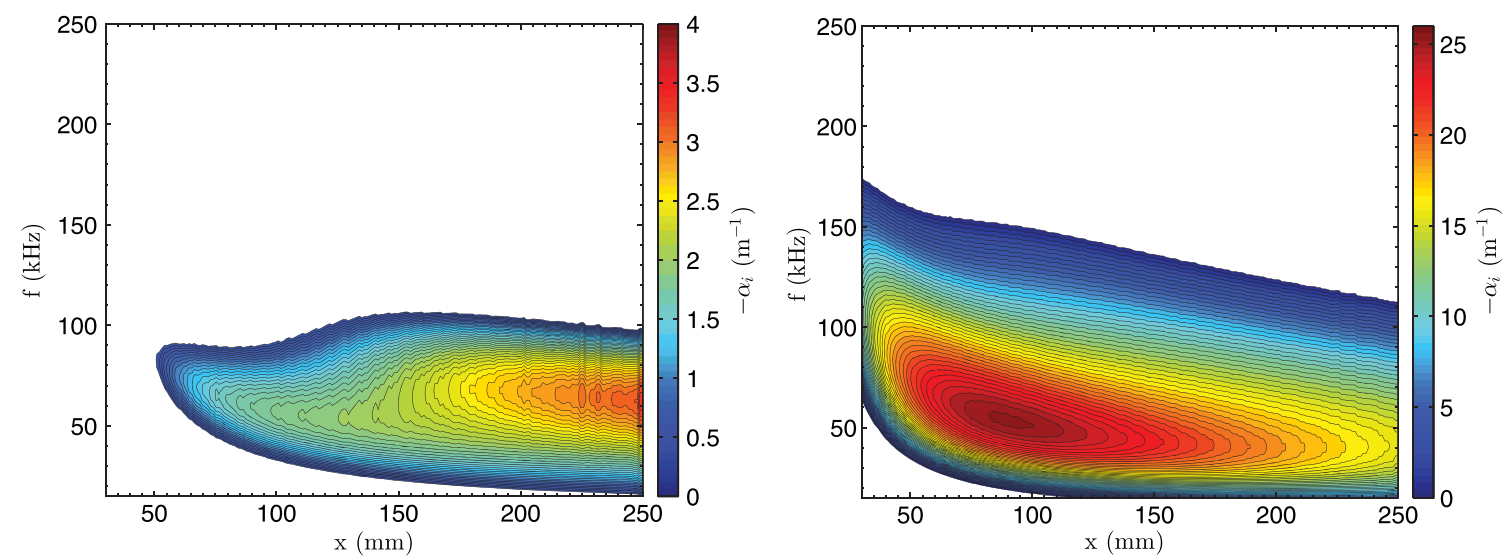

Fig. 19 Contours of spatial growth rate versus streamwise distance and frequency for the baseline case of a cone with no injection. Left, 2-D waves; right, 3-D waves with $\beta=290$.

boundary condition on the temperature fluctuation, $\hat{\theta}=0$, is satisfied when an eigenvalue $\alpha$ has been found. The complex eigenvalue search is conducted using the secant method with a convergence tolerance of $10^{-5}$. The code used to perform the stability analysis has been validated by reproducing the results of several other researchers for flat-plate boundary layers under hypersonic, perfect gas conditions $[\underline{4}, \underline{23}, \underline{27}, \underline{28}]$.

In this paper the stability analysis is locally parallel, meaning that streamwise gradients in the mean flow are ignored, as is the wallnormal velocity. The neglect of the wall-normal velocity is reason-
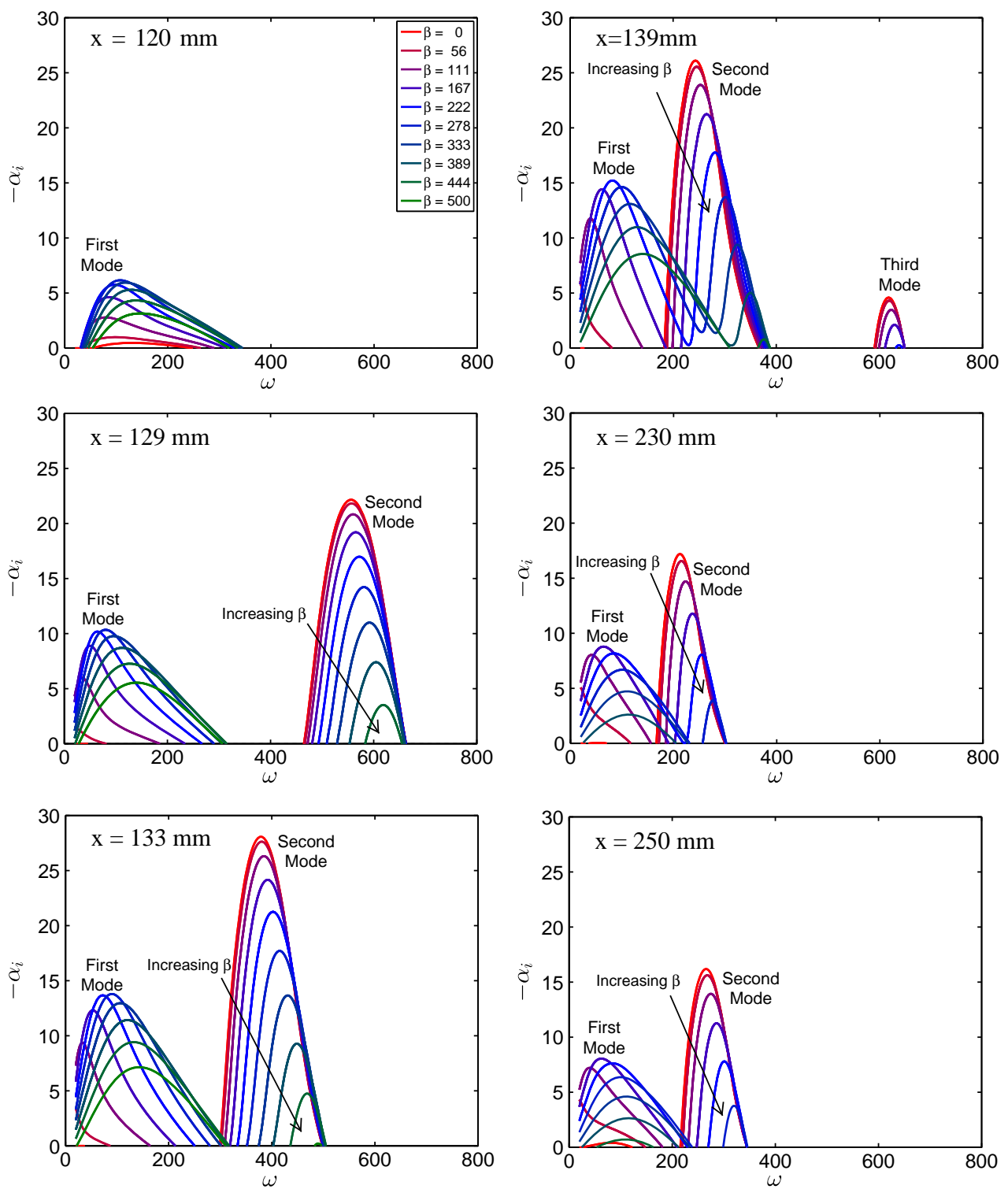

Fig. 20 Stability diagrams for a cylindrical injector with $\boldsymbol{m}=\mathbf{0 . 6}$. Each individual curve corresponds to a different spanwise wavenumber $\beta$. 


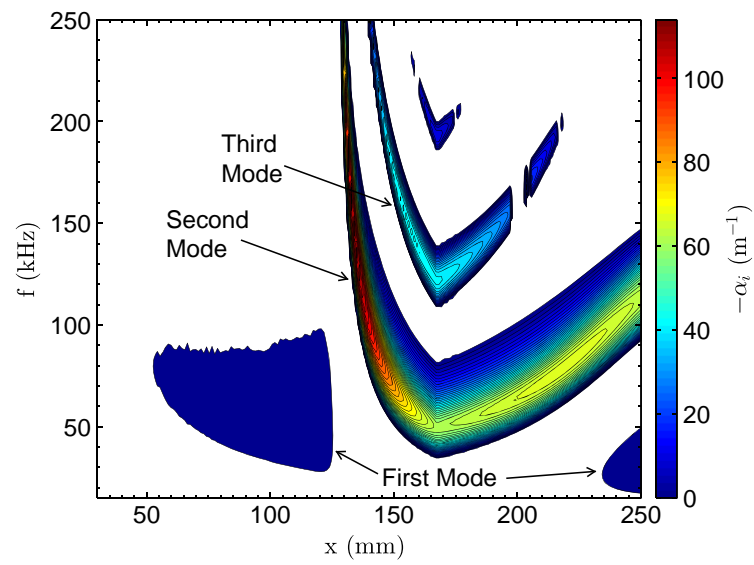

a)

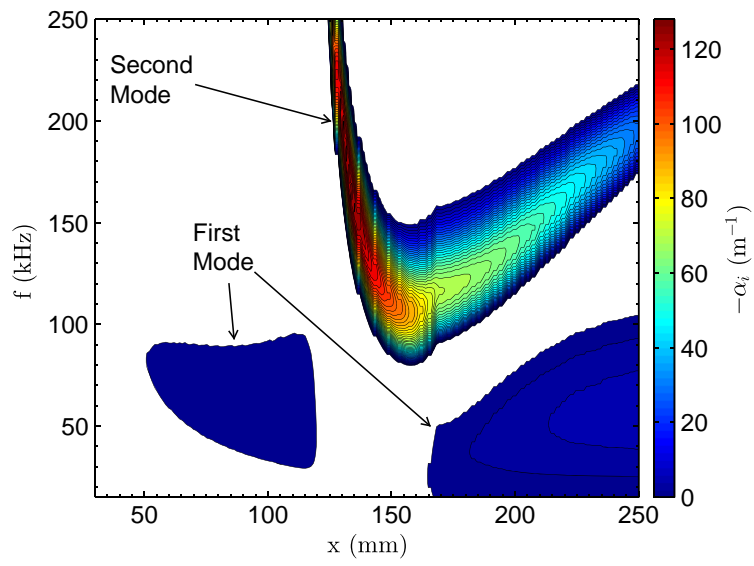

c)

Fig. 21 Contours of spatial growth rate versus streamwise distance and frequency for $m=0.6$ : a) cylindrical injector, 2-D waves; b) cylindrical injector, 3-D waves with $\beta=222$; c) conical injector, 2-D waves; and d) conical injector, 3-D waves with $\beta=278$.

able because the injection velocity $(<20 \mathrm{~m} / \mathrm{s})$ is less than $3 \%$ of the freestream velocity. Likewise, for a conical injector the wall-normal component of velocity in the freestream above the injector (caused by the change in slope of the wall) is only $8.7 \%$ of the tangential velocity component. However, the parallel flow approximation is questionable in the injection region because the mean flow varies strongly in the streamwise direction. Likewise, the assumption of a planar boundary layer may be questionable because the thickness of the boundary layer over the injector $(3-5 \mathrm{~mm})$ is not much smaller than the $11 \mathrm{~mm}$ radius of the injector (c.f. Fig. 8). A further difficulty is that, for the cylindrical injector, the slope of the wall is discontinuous at the beginning and end of the injector. Because the linear stability analysis is carried out on wall-normal planes, the flow profiles used in

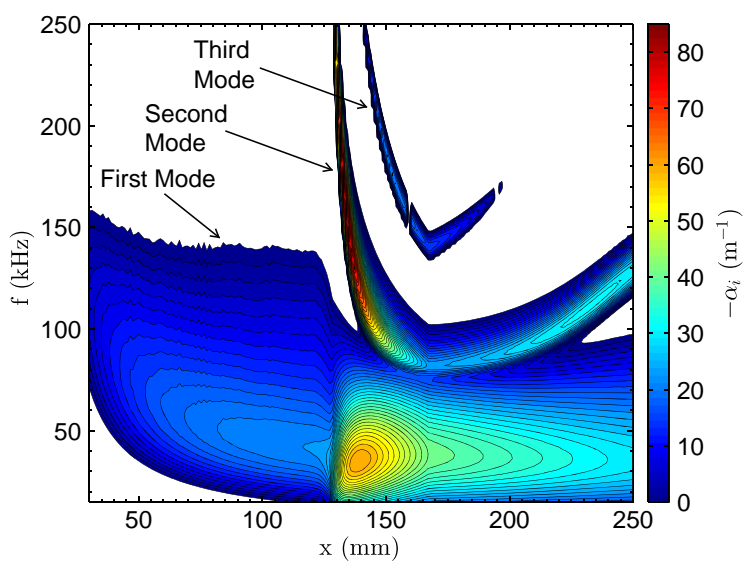

b)

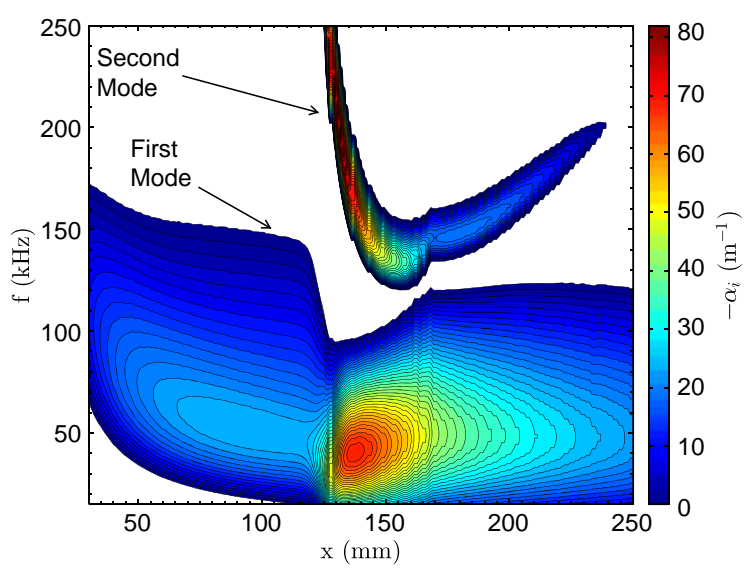

d)

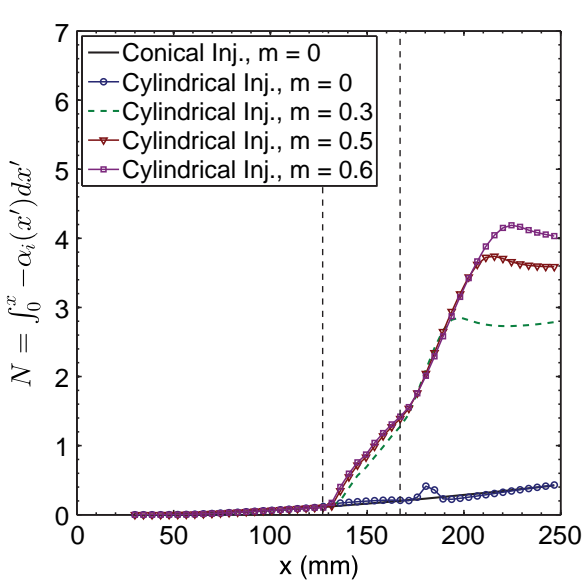

the stability analysis are discontinuous at these locations, and likewise the growth rates can be discontinuous. All of these factors are likely to quantitatively affect the disturbance growth rates and are deserving of further investigation. Nevertheless, the present method produces results that are in reasonably good agreement with the experiments and furnishes some insight regarding the influence of injection on the stability of the boundary layer.

Figure 19 shows contours of the spatial growth rate $-\alpha_{i}$ against streamwise distance and frequency for a cone with no injection. This provides a reference configuration against which the cases with gas injection can be compared. The diagram on the left contains 2-D waves, while the diagram on the right contains 3-D waves with spanwise wavenumber $\beta=290$, which is the wavenumber that

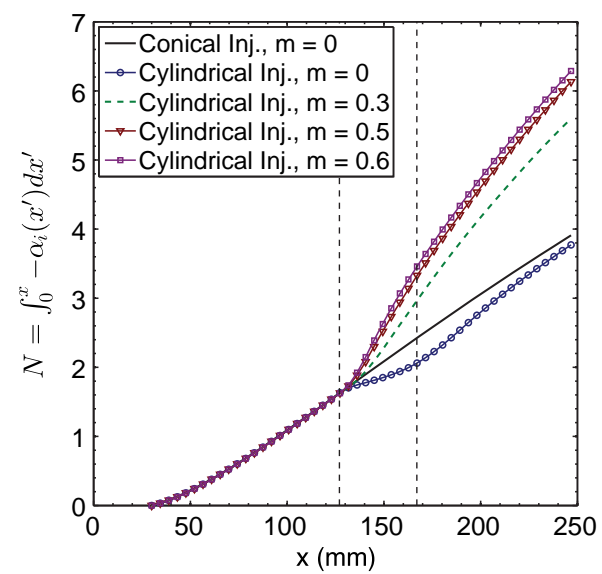

Fig. 22 Maximum $N$ factors for various injection rates. Left, 2-D waves; right, 3-D waves with $\beta=220$. 
produces the largest $N$ factors for $0<x<0.25 \mathrm{~m}$. The unstable regions in both figures correspond to first-mode waves, while the second mode remains stable at these conditions. The flow is nominally at Mach 4, with a postshock Mach number of about 3.8, which is very close to the point at which the second mode begins to be unstable according to the adiabatic stability calculations of Mack [4]. In this experiment, however, the wall temperature of $300 \mathrm{~K}$ is slightly above the adiabatic temperature, which further stabilizes the second mode so that it is completely stable for the Reynolds numbers relevant to the experiment.

Next the effect of gas injection is considered. Examples of dispersion curves for the cylindrical injector with $m=0.6$ are shown in Fig. 20. Each dispersion curve contains several different spanwise wavenumbers between 0 and 500 (nondimensionalized by the cone length $\left.L_{c}\right)$. Upstream of the injector $(x=120 \mathrm{~mm})$, only the first mode is unstable and the spatial growth rate is rather small. Slightly downstream of the leading edge of the injector $(x=129 \mathrm{~mm})$, the second mode also becomes unstable and its growth rate surpasses that of the first mode. Further downstream, the frequencies of the unstable modes shift to lower values because of the increasing thickness of the injection layer, and the third mode also becomes unstable (upperright frame of Fig. 20). Finally, downstream of the injector the flow profile relaxes back toward that of an undisturbed boundary layer and the stability characteristics do likewise. However, even at the end of the cone the flow profiles have not completely relaxed and the second mode remains unstable. The dispersion curves for the conical injector are qualitatively similar to those shown in Fig. 20. However, because the "dead flow" region is thicker for a cylindrical injector than for a conical one (see Fig. 17), the second- and third-mode growth rates are larger for the cylindrical case.

Figure 21 presents the stability diagram in contour form, which clarifies the streamwise variation of the unstable regions. The top and bottom rows show the growth rates for cylindrical and conical injectors, respectively, both at an injection rate of $m=0.6$. The left column reports the amplification rates for $2-\mathrm{D}$ waves, while the right column represents the 3-D waves that feature the largest first-mode growth rate. For both injector geometries, the 2-D stability diagram (left) is dominated by second and higher instability modes with very little contribution from the first mode. However, for 3-D waves (right) the first mode contributes a broad unstable region. Although the maximum growth rate of the first mode is not as large as that of the second mode, the first mode is unstable over a much longer streamwise distance.

For the first mode, the most unstable frequency remains nearly constant along the cone length. In contrast, the second- and thirdmode frequencies drop sharply over the injector (127-167 mm) and then rise again downstream of the injector, varying inversely with the injection layer thickness (see Fig. 18). This behavior has a major impact on the growth of instability waves. Second- and third-mode wave packets of a given frequency that are propagating downstream are only unstable over a short distance, and so their net amplification is rather small. In contrast, first-mode wave packets are unstable over a much longer distance and experience larger total amplification.

This fact is made quantitative by computing $N$ factors, that is, horizontal integrals across the stability diagrams in Fig. 21. The $N$ factors are determined according to the formula

$$
N(x, \omega, \beta)=\int_{x_{o}}^{x}-\alpha_{i}(\xi, \omega, \beta) \mathrm{d} \xi
$$

where $x_{o}(\omega, \beta)$ is the location at which the wave first becomes unstable. Separate $N$-factor curves are computed for each frequency $\omega$ and spanwise wavenumber $\beta$. For each condition $(x, \omega, \beta)$, the stability analysis may find more than one discrete mode, and the $N$ factor integral is carried out using the envelope over all of these modes; that is, the most unstable or least stable value of $-\alpha_{i}$ is used in computing the integral of Eq. (8). Although in this work the spanwise wavenumber $\beta$ is held constant during the calculation of the $N$-factor integral, it may be more meaningful to hold the product $\beta r_{c}(x)$ constant during the integration, where $r_{c}(x)$ is the local cone radius. This approach has also been tested and has been found not to signi- ficantly affect the maximum $N$ factors or the qualitative behavior of any of the results that follow. Figure 22 shows the envelope of the $N$ factor curves over all frequencies for several different injection rates. The left diagram in this figure presents the $N$-factor distribution for 2D waves, while the right diagram is for 3-D waves with $\beta=220$, which is the value that produced the largest $N$ factors at the higher injection rates. For reference, the $N$ factors of a plain cone with no injection are also included. Clearly, the $N$ factors are much larger for the 3-D waves than for the 2-D ones, indicating that the first-mode instability plays an important role in the transition process. This possibility has not been explored in previous computational studies of boundary-layer transition with gas injection. For the cylindrical injector with no injection, the 3-D waves are slightly stabilized relative to the plain cone. This is consistent with the finding of Malik [29] that favorable pressure gradients tend to stabilize the first mode.

The most amplified wavenumbers from the stability analysis can be compared with the values determined experimentally. These wavenumbers were extracted from the schlieren photographs in Sec. IV.A and the resulting distribution of spectral power versus wavenumber was given in Fig. 13. Here a similar analysis is performed using the linear stability results by plotting the $N$ factor vs wavenumber, as shown in Fig. 23. Each line in this diagram corresponds to a different streamwise location $x$, with the disturbance frequency parameterizing the curves. The results shown are for a 3-D wave with $\beta=220$, which was the spanwise wavenumber that resulted in the largest $N$ factor at the end of the cone.

The taller peak in Fig. 23 corresponds to first-mode waves, which grow monotonically in amplitude and decrease slightly in wavenumber as $x$ increases. The second peak at about $1 / \lambda=0.16 / \mathrm{mm}$ corresponds to second-mode waves, which grow mainly over the injector and slightly downstream from it, but stop growing farther downstream. By comparing Figs. 13 and 23, it is found that the most unstable wavelength $(13.1 \mathrm{~mm})$ from stability analysis is considerably larger than that of the experiments $(6.8 \mathrm{~mm})$, but the wavelength of the 2-D second-mode waves $(6.25 \mathrm{~mm})$ is quite close to the experimental value. This discrepancy may be the result of the experimental technique. Schlieren images are integrated in the spanwise direction and may be less sensitive to the oblique first-mode waves than to 2-D waves. It is more likely, however, that the discrepancy is due to limitations on the linear stability analysis because of the assumptions made. Nonparallel effects are ignored in this analysis, and the most amplified streamwise wavelength of $13.1 \mathrm{~mm}$ is about one third the length of the injector and is clearly not small compared with the length scale over which the mean flow is changing. Additionally, the spanwise wavelength of the first-mode waves $(7 \mathrm{~mm})$ is not negligible compared with the circumference of the cone downstream of the injector, which is on the order of $100 \mathrm{~mm}$. In the physical flow this could inhibit the development of periodic oblique waves as they would have to wrap three-dimensionally around the cone.

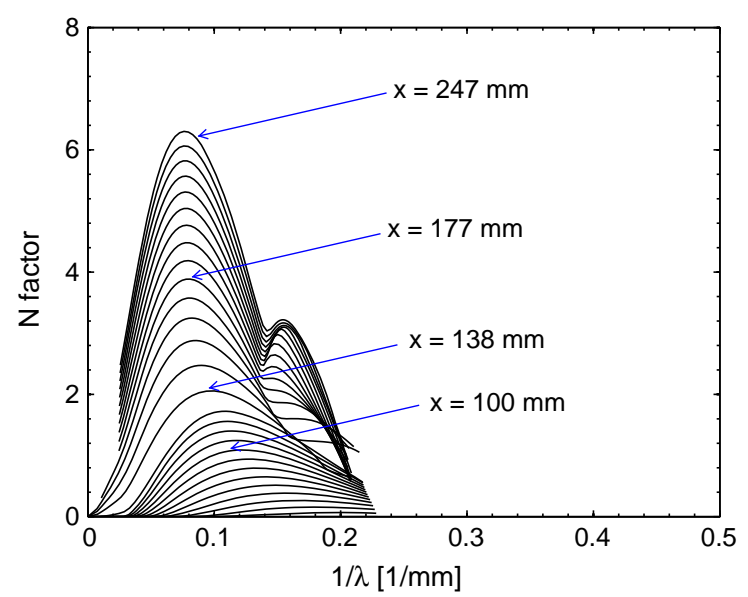

Fig. 23 Maximum $N$ factor as a function of wavenumber for the cylindrical injector, $m=0.6, \beta=220$. Compare to Fig. $\underline{13}$. 
Transition Reynolds numbers can be predicted using the semiempirical $e^{N}$ method in which transition is correlated with a particular value of the $N$ factor. Figure 9 compares the experimentally measured transition locations with those predicted by the linear stability theory for $N=5$ and 6 . The measured data match the prediction for $N=5$ quite well. Transition locations for $N=6$ are also shown to give an indication of the sensitivity of transition distance to the choice of $N$. For all injection rates, the maximum growth is experienced by $3-\mathrm{D}$ waves with spanwise wavenumbers in the range of $\beta=200-300$, which correspond to spanwise wavelengths of 5-7 mm. The amplification of 2-D waves is considerably smaller: for injection rates of $0<m<1$, the 2-D $N$ factor is less than 4 at the measured transition location, which suggests that the second mode is not responsible for the observed instability.

\section{Conclusions}

An experimental study was performed to examine the effects of injecting gas into the boundary layer on a slender body in supersonic flow. Of particular interest is the stability of the injection layer formed downstream of injection and whether through shaping the injector a stable flow could be created that would allow a vibrationally active gas such as carbon dioxide to stabilize a hypervelocity boundary layer. The present study does not consider effects of vibrational nonequilibrium but focuses only on the fluid mechanics of the flow created by boundary-layer injection. Injector shaping helps to minimize waves formed by injection, but injection still creates an unstable layer near the wall of the body.

Experiments were performed in the Mach 4 Ludwieg tube at Caltech using a 5-deg half-angle cone equipped with either a conical injector or a cylindrical injector at a variety of injection mass flow rates. Injection is found to form a strong oblique shock wave that propagates into the inviscid region of the flow when the conical injector is used, and the resulting pressure jump across the shock is believed to cause rapid transition to turbulence in the injection layer. When the cylindrical injector is used, there is a "tuned" injection rate that minimizes the strength of the oblique wave, and at other mass flow rates the strength of the wave is significantly lessened compared with that of the wave formed using the conical injector. Transition does not occur immediately when the cylindrical injector is used; rather, transition occurs downstream of the injector with higher mass flow rates producing earlier transition. It is possible to determine the wavelength of the instability waves in the injection layer using a highspeed schlieren visualization technique, and the wavelength for the "tuned" case nearly matches the prediction for that of 2-D secondmode waves. However, the wavelength of the instability waves does not scale with the thickness of the injection layer.

Our computational results suggest that the instability is dominated by oblique first-mode waves, which have not been considered in other injection studies at higher Mach numbers and larger flow enthalpies. There is also an unstable second mode in the linear stability analysis, but $N$ factors for the first-mode waves are higher. Thus, it is expected that the first mode is primarily responsible for transition in this flow. The observation of large-scale structures in the flow downstream of injection in the experiments suggests a substantial cross-stream coherence and the measured wavelength of the instability waves in the experiment agrees well with that of the second-mode waves in the linear stability analysis. This is likely due to the limitations of the computational analysis where the flow is assumed to be locally parallel, which is not the case near the injector, and the thickness of the injection layer is small compared with the cone radius, which is not true for this flow. The wavelength of the instability waves in the experiment does not appear to scale with the layer thickness, which is not true for second-mode waves in a boundary layer. It is possible that transition in this flow may be caused by a 2-D Kelvin-Helmholtz instability.

Clearly, the stability characteristics of supersonic flow with injection are complex and worthy of further study. These are the first quantitative measurements of the instability waves in such a flow [17]. An experimental campaign to make more in-depth quantitative measurements is currently underway to better characterize the stabil- ity properties of this flow. The use of injection for delaying boundarylayer transition appears to be limited because of the instability presented here, but because turbulence is desired in scramjet inlets injection in supersonic flow remains a highly relevant research topic.

\section{Acknowledgments}

This work was sponsored in part by the Air Force Office of Scientific Research (AFOSR) and NASA through the National Center for Hypersonic Research in Laminar-Turbulent Transition and also by AFOSR award number FA9550-10-1-0491. The views expressed herein are those of the authors and should not be interpreted as necessarily representing the official policies or endorsements, either expressed or implied, of AFOSR or the U.S. Government. The authors would like to thank Nicholaus Parziale and Bahram Valiferdowsi for guidance and assistance in the laboratory. The authors would additionally like to acknowledge Joseph Jewell, Ivett Leyva, and Ross Wagnild for previous work done on this topic. Finally, the authors thank Alexander Fedorov for the concept of using a negative slope on the cone surface to compensate for injection. Without this key idea, this work would not have been performed.

\section{References}

[1] Barth, J. E., Wheatley, V., and Smart, M. K., "Hypersonic Turbulent Boundary-Layer Fuel Injection and Combustion: Skin-Friction Reduction Mechanisms," AIAA Journal, Vol. 51, No. 9, Sept. 2013, pp. 2147-2157. doi:10.2514/1.J052041

[2] Berry, S. A., Auslender, A. H., Dilley, A. D., and Calleja, J. F., "Hypersonic Boundary-Layer Trip Development for Hyper-X," Journal of Spacecraft and Rockets, Vol. 38, No. 6, Nov.-Dec. 2001, pp. 853864.

doi: $10.2514 / 2.3775$

[3] Jewell, J. S., Leyva, I. A., Parziale, N. J., and Shepherd, J. E., "Effect of Gas Injection on Transition in Hypervelocity Boundary Layers," 28th International Symposium on Shock Waves, Springer, Berlin, 2012, pp. 735-740.

[4] Mack, L. M., "Boundary-Layer Linear Stability Theory,” Jet Propulsion Laboratory and California Inst. of Technology TR AD-P004 046 , Pasadena, CA, 1984.

[5] Fedorov, A. V., "Transition and Stability of High-Speed Boundary Layers," Annual Review of Fluid Mechanics, Vol. 43, No. 1, 2011, pp. 79-95. doi:10.1146/annurev-fluid-122109-160750

[6] Fujii, K., and Hornung, H. G., "A Procedure to Estimate the Absorption Rate of Sound Propagating Through High Temperature Gas," California Inst. of Technology TR FM-2001.004, Pasadena, CA, 2001.

[7] Wagnild, R. M., Candler, G. V., Leyva, I. A., Jewell, J. S., and Hornung, H. G., "Carbon Dioxide Injection for Hypervelocity Boundary Layer Stability," 48th Aerospace Sciences Meeting, AIAA Paper 2009-1287, 2009.

[8] Leyva, I. A., Jewell, J. S., Laurence, S., Hornung, H. G., and Shepherd, J. E., "On the Impact of Injection Schemes on Transition in Hypersonic Boundary Layers," 16th AIAA International Space Planes and Hypersonic Systems and Technologies Conference, AIAA Paper 20097204, 2009.

[9] Fedorov, A. V., Malmuth, N. D., Rasheed, A., and Hornung, H. G., "Stabilization of Hypersonic Boundary Layers by Porous Coatings," AIAA Journal, Vol. 39, No. 4, 2001, pp. 605-610. doi:10.2514/2.1382

[10] Fedorov, A. V., Soudakov, V. G., and Leyva, I. A., "Stabilty Analysis of High-Speed Boundary-Layer Flow with Gas Injection," AIAA 7th Theoretical Fluid Mechanics Conference, AIAA Paper 2014-1271, June 2014

[11] Mouton, C. A., "Transition between Regular Reflection and Mach Reflection in the Dual-Solution Domain,’ Ph.D. Thesis, California Inst. of Technology, Pasadena, CA, 2007.

[12] Parziale, N. J., Damazo, J. S., Schmidt, B. E., Wang, P. S., Hornung, H. G., and Shepherd, J. E., "Pulsed Laser Diode for Use as a Light Source for Short-Exposure, High-Frame-Rate Flow Visualization," AIAA SciTech 2015, AIAA, Reston, VA, 2015.

[13] Schmidt, B. E., "Compressible Flow Through Porous Media with Application to Injection," California Inst. of Technology IR-FM2014.001, Pasadena, CA, 2014.

[14] Frederking, T. H. K., Hepler, W. A., Yuan, S. W. K., and Feng, W. F., "Determination of the Darcy Permeability of Porous Media Including 
Sintered Metal Plugs," Advances in Cryogenic Engineering, 1986, pp. 505-515.

[15] Pappas, C. C., and Okuno, A., "Measurements of Skin Friction of the Compressible Turbulent Boundary Layer on a Cone with Foreign Gas Injection," Journal of the Aero/Space Sciences, Vol. 27, No. 5, May 1960, pp. 321-333. doi: $10.2514 / 8.8533$

[16] Pappas, C. C., and Okuno, A., "Heat-Transfer Measurement for Binary Gas Laminar Boundary Layers with High Rates of Injection," NASA TN-D-2473, 1964

[17] Schneider, S. P., "Hypersonic Boundary-Layer Transition with Ablation and Blowing," Journal of Spacecraft and Rockets, Vol. 47, No. 2, March-April 2010, pp. 225-237. doi:10.2514/1.43926

[18] Li, F., Choudhari, M., Chang, C.-L., and White, J., "Effects of Injection on the Instability of Boundary Layers over Hypersonic Configurations," Physics of Fluids, Vol. 25, No. 10, 2013, Paper 104107. doi:10.1063/1.4825038

[19] Johnson, H., Seipp, T., and Candler, G., "Numerical Study of Hypersonic Reacting Boundary Layer Transition on Cones," Physics of Fluids, Vol. 10, No. 10, Oct. 1998, pp. 2676-2685. doi:10.1063/1.869781

[20] Wright, M., Candler, G., and Bose, D., "Data-Parallel Line Relaxation Method for the Navier-Stokes Equations," AIAA Journal, Vol. 36, No. 9, Sept. 1998, pp. 1603-1609. doi: $10.2514 / 2.586$

[21] Johnson, H. B., "Thermochemical Interactions in Hypersonic Boundary Layer Stability," Ph.D. Thesis, Univ. of Minnesota, Minneapolis, MN, July 2000.

[22] Mack, L. M., "Computations of the Stability of the Laminar Compressible Boundary Layer," Methods in Computational Physics, edited by Alder, B., Fernback, S., and Rotenberg, M., Vol. 4, Academic Press, New York, 1965, pp. 247-299.

[23] Malik, M. R., "Numerical Methods for Hypersonic Boundary Layers," Journal of Computational Physics, Vol. 86, No. 2, 1990, pp. 376-413. doi:10.1016/0021-9991(90)90106-B

[24] Conte, S., "The Numerical Solution of Linear Boundary Value Problems," SIAM Review, Vol. 8, No. 3, July 1966, pp. 309-321. doi: $10.1137 / 1008063$

[25] Garg, V., "Improved Shooting Techniques for Linear Boundary Value Problems," Computer Methods in Applied Mechanics and Engineering, Vol. 22, No. 1, April 1980, pp. 87-99. doi:10.1016/0045-7825(80)90052-3

[26] Davey, A., "An Automatic Orthonormalization Method for Solving Stiff Boundary-Value Problems," Journal of Computational Physics, Vol. 51, No. 2, Aug. 1983, pp. 343-356. doi:10.1016/0021-9991(83)90098-0

[27] Fedorov, A., and Tumin, A., "High-Speed Boundary-Layer Instability: Old Terminology and a New Framework," AIAA Journal, Vol. 49, No. 8 , Aug. 2011, pp. 1647-1657. doi:10.2514/1.J050835

[28] Ma, Y., and Zhong, X., "Receptivity of a Supersonic Boundary Layer over a Flat Plate. Part 1. Wave Structures and Interactions," Journal of Fluid Mechanics, Vol. 488, 2003, pp. 31-78. doi:10.1017/S0022112003004786

[29] Malik, M. R., "Prediction and Control of Transition in Supersonic and Hypersonic Boundary Layers," AIAA Journal, Vol. 27, No. 11, Nov. 1989 , pp. 1487-1493. doi: $10.2514 / 3.10292$ 